\title{
Exosomal miR-106b-5p derived from melanoma cell promotes primary melanocytes epithelial-mesenchymal transition through targeting EphA4
}

Wenkang Luan ${ }^{1 *+}$, Yuting Ding ${ }^{2+}$, Haolan $\mathrm{Xi}^{3+}$, Hongru Ruan ${ }^{1}$, Feng $\mathrm{Lu}^{1}$, Shaojun Ma ${ }^{1}$ and Jinlong Wang ${ }^{1}$

\begin{abstract}
Background: Cancer-secreted exosomal miRNAs regulates the biological processes of many tumours. The serum level of exosomal miR-106b-5p is significantly increased in melanoma patients. However, the role and molecular mechanisms of exosomal miR-106b-5p in melanoma remains unclear.

Methods: Quantitative real-time polymerase chain reaction (qRT-PCR) was used to detect the expression of miR106b-5p and EphA4 in melanoma tissues. Transmission electron microscopy (TEM) and western blotting were used to identify exosome. QRT-qPCR and Cy3-labelled miR-106b-5p were used to demonstrated the transmission of melanoma cell-secreted exosomal miR-106b-5p. Western blotting, Immunofluorescence, adhesion, transwell and scratch wound assay were used to explore the role of exosomal miR-106b-5p in melanocytes. Luciferase reporter assays and RNA-Chromatin Immunoprecipitation (ChIP) assay were used to confirm whether erythropoietinproducing hepatocellular carcinoma receptor A4 (EphA4) was a direct target of miR-106b-5p.
\end{abstract}

Results: We found that miR-106b-5p levels were increased in melanoma tissue, and high miR-106b-5p expression is an independent risk factor for the overall survival of patients with melanoma. miR-106b-5p is enriched in melanoma cell-secreted exosomes and transferred to melanocytes. Exosomal miR-106b-5p promotes the epithelial-tomesenchymal transition (EMT), migration, invasion and adhesion of melanocytes. Exosomal miR-106b-5p exerted its role by targeting EphA4 to activate the ERK pathway. We demonstrated that exosomal miR-106b-5p promoted melanoma metastasis in vivo through pulmonary metastasis assay.

Conclusions: Thus, melanoma cell-secreted exosomal miR-106b-5p may serve as a diagnostic indicator and potential therapeutic target in melanoma patients.

Keywords: Melanoma, Exosomal, miR-106b-5p, EphA4, EMT

\footnotetext{
* Correspondence: luanwenkang@126.com

'Wenkang Luan, Yuting Ding and Haolan Xi contributed equally to this work. 'Department of Plastic Surgery, Affiliated People's Hospital of Jiangsu University, 8 Dianli Road, Zhenjiang 212000, Jiangsu, China

Full list of author information is available at the end of the article
} 


\section{Background}

Malignant melanoma is the most aggressive skin cancer and its global incidence is increasing every year [1-3]. It has been reported to be the main cause of skin tumourrelated death [4]. Although surgery is the first treatment for the primary stage of melanoma, the survival of patients with metastatic dissemination is significantly reduced [5]. Therefore, it is important to understand the molecular mechanisms underlying melanoma progression and metastasis.

Exosomes, which are $30-150 \mathrm{~nm}$ endocytic vesicles, are produced and released by a variety of cells, including tumour cells $[6,7]$. Exosomes have been implicated in intercellular communication by transmitting different molecules, including proteins, lipids, and functional RNA molecules, from donor cells to recipient cells [8, 9]. miRNAs, which are 20-22 nucleotide non-coding RNA, regulate gene expression by cleaving or inhibiting the translation of target mRNAs [10, 11]. Cancersecreted exosomal miRNAs can be transferred into recipient cells to regulate target genes and biological processes of tumours $[12,13]$. Melanoma cells release a large number of exosomes, and substantial differences in the profiles of exosomal miRNAs in the plasma of patients with melanoma have been observed [14, 15]. Nevertheless, only a few exosomal miRNA functions have been identified in melanoma [12, 16]. It has been reported that exosomal miR-106b-5p in serum is present at significantly higher levels in patients with melanoma [16]. However, the function and molecular mechanisms of exosomal miR-106b-5p during melanoma development remains unknown.

Melanoma is a cancer that originates from melanocytes. Although melanocytes do not belong to the epithelial cell line, primary melanocytes express E-cadherin [17]. Normal melanocytes also express epithelial-tomesenchymal transition (EMT)-inducing transcription factors (EMT-TFs), which are intrinsic factors that lead to the high metastatic propensity of melanoma [17]. The EMT process contributes to the progression and metastasis of melanoma [18], but there are few studies on the effect of melanoma exosomes on the progression of melanoma by affecting EMT in tumour microenvironment. EphA4, which is a member of the large Eph (erythropoietin-producing hepatocellular carcinoma) family of receptor tyrosine kinases, has been shown to play oncogenic and tumour-suppressive roles in many human tumours $[19,20]$. EphA4 effectively suppresses the EMT and metastatic capabilities of melanoma cells by interfering with the activation of ERK [21].

In this study, we showed that miR-106b-5p expression is increased in melanoma tissue and that high miR106b-5p expression is an independent risk factor for patients with melanoma. Moreover, miR-106b-5p was also shown to be highly enriched in exosomes secreted from melanoma cells. Melanoma cell-derived exosomal miR106b-5p promotes melanocyte EMT by directly repressing EphA4 to activate ERK pathways, leading to the establishment of a tumour metastasis-supporting microenvironment. Thus, exosomal miR-106b-5p derived from melanoma cells may serve as a diagnostic indicator and potential therapeutic target in patients with melanoma.

\section{Materials and methods \\ Human tissues}

We collected 36 primary malignant melanoma tissues and adjacent normal tissues from the Affiliated People's Hospital of Jiangsu University. Samples were stored in liquid nitrogen after collection, and the clinicopathological features of the tissue were independently diagnosed by two pathologists. All patients received no chemotherapy or radiotherapy before operation. Serum samples of peripheral blood from patients with primary and 12 metastatic melanoma were also collected in the Affiliated People's Hospital of Jiangsu University. This study protocol was approved by the Human Research Ethics Committee of the Affiliated People's Hospital of Jiangsu University. The informed consent was obtained from each participant. Melanoma cases from The Cancer Genome Atlas (TCGA) and the Gene Expression Omnibus (GEO\#GSE34460 and GEO\#GSE24996) were also included in this study.

\section{Cell culture}

The A375 human malignant melanoma cell line was obtained from the Chinese Academy of Sciences Cell Bank (Shanghai, China), and the A2058, SK-MEL-1 and SKMEL-28 human malignant melanoma cell lines were obtained from the American Type Culture Collection. The cells were cultured in Dulbecco's modified Eagle's medium (DMEM; Gibco, USA), which contained 10\% foetal bovine serum (FBS; Invitrogen, USA). HEMa-LP human epidermal melanocytes were purchased from Invitrogen and grown in medium 254 (Cascade Biologics).

\section{Oligonucleotides, plasmids and transfection}

miR-106b-5p mimic, miR-106b-5p inhibitor and negative controls were chemically synthesized by GenePharma (Shanghai, China), and the small interference RNAs (siRNAs) for the reduceion of EphA4 expression were also obtained from GenePharma. EphA4 was amplified in full length and inserted into pcDNA3.1 vectors (Invitrogen, USA) to construct EphA4 plasmid. The lentiviral empty vector and miR-106b-5p inhibitor vector were also obtained from GeneChem (Shanghai, China). Melanoma cells were infected with lentiviruses in order to obtain stably expressing miR-106b-5p inhibitor cells. 
The oligonucleotides and plasmids were transfected into melanoma cells or melanocytes via Lipofectamine 2000 (Invitrogen, USA) according to the manufacturer's instructions.

\section{Extraction of RNA and quantitative RT-PCR}

Total RNA was extracted from cells and tissues by using TRIzol reagent (Invitrogen, USA). Reverse transcription (RT) was performed using Fermentas reverse transcription reagents and an Applied Biosystems ${ }^{\circ}$ TaqMan $^{\circ}$ MicroRNA Reverse Transcription Kit (Applied Biosystems, CA). An ABI StepOnePlus system (Applied Biosystems, CA) was used for amplification reactions according to the predetermined conditions. To analyse the expression of miR-106b-5p, the primer for miR-106b-5p was purchased from RiboBio (Guangzhou, China), and U6 was used for normalization. The primer for miR106b-5p were 5' -TGCGGCAACACCAGTCGA TGG-3' and 5 '-CCAGTGCAGGGTCCGAGGT-3'. The primer for U6 were 5' -CTCGCTTCGGGCAGCACA-3' and 5' AACGCTTCACGAATTTGCGT-3'. To analyse the expression levels of EphA4, GAPDH was used for normalization. The primers for EphA4 were 5'CAGAGGTAAGGGTAGGAGGC-3' and $5^{\prime}$-AGCAGT GTAGCGAGCACAAC-3'. GAPDH forward, 5'-AACT TTGGCATTGTGGAAGG-3' and reverse, 5'-GGAT GCAGGGATGATGTTCT-3'. The data were calculated using the $2^{-\Delta \Delta \mathrm{Ct}}$ method.

\section{Western blot analysis}

Total protein from cells, tissues and exosomes was extracted with RIPA buffer (Kengen, China) and quantitatively analysed with a BCA Protein Assay Kit (Beyotime, China). Western blotting was conducted as previously described [22]. Antibodies against CD63, TSG101, calnexin, fibronectin, EphA4, Snail, total ERK, and $\mathrm{p}$-ERK ${ }^{\mathrm{T} 202 / \mathrm{Y} 204}$ were purchased from Abcam (Cambridgeshire, UK). Antibodies against E-cadherin, $\mathrm{N}$-cadherin, $ß$-actin and GAPDH were purchased from Cell Signalling Technology (CST, USA).

\section{Luciferase reporter assay}

The 3'-UTR fragment of EphA4 containing the binding site of miR-106b-5p was cloned into the pMIR-REPORT vector, and mutant plasmids were used as a control. The miR-106b-5p mimic and related reporter plasmids were co-transfected into melanocytes. A Dual Luciferase Reporter Assay Kit (Promega, USA) was used to analyse the luciferase activity after $48 \mathrm{~h}$ according to the manufacturer's instructions.

\section{Isolation of RISC-associated RNA}

HEMa-LP cells overexpressing miR-106b-5p or NC were fixed with $1 \%$ formaldehyde, and chromatin fragments were then processed. The cells were lysed in NETN buffer and incubated with Dynabeads Protein A (Invitrogen, USA) supplemented with IgG or the anti-Pan-Ago clone 2A8 antibody (Millipore, USA). Immunoprecipitated RNA was released by proteinase $\mathrm{K}$ digestion. Phenol/ chloroform/isopropyl alcohol was used to extract RNA. RNA was purified by glycogen ethanol precipitation, resolved and treated with DNase I.

\section{Exosome isolation, labelling and identification}

Malignant melanoma cells were cultured in DMEM supplemented with 10\% exosome-depleted FBS for $48 \mathrm{~h}$. Ten millilitres of cell conditioned medium or $250 \mu \mathrm{l}$ serum (peripheral blood from patients with primary and metastatic melanoma) was mixed with ExoQuick exosome precipitation solution (System Biosciences, USA). Exosome isolation was conducted using ExoQuick-TC ${ }^{\mathrm{mm}}$ (System Biosciences, USA) according to the manufacturer's protocol. For transmission electron microscopy, the exosomes were loaded onto carbon-coated 300 mesh copper grids (Agar Scientific Ltd., Stansted, UK), fixed with $3 \%$ glutaraldehyde and $1 \%$ osmium tetroxide, and then stained with uranyl acetate and lead citrate. The exosomes were observed by Transmission electron microscopy (TEM, Hitachi, Japan) after air-drying. For RNA extraction from the exosomes, a miRNeasy Mini Kit (Qiagen) was used. The concentration of exosomes protein was determined by using a BCA Protein Assay Kit (Beyotime, China). A PKH67 Green Fluorescent Cell Linker Mini Kit (Sigma, USA) was used to label the purified exosomes according to the manufacturer's instructions. Images were acquired with a confocal microscope.

\section{Immunofluorescence (IF) assay}

HEMa-LP cells were seeded on collagen-coated glass coverslips and fixed in $4 \%$ formaldehyde after washing with phosphate-buffered saline (PBS) at the time of cell harvest. The cells were permeabilized with $0.1 \%$ Triton $\mathrm{X}-100$ and blocked with $1 \%$ BSA in PBS to prevent nonspecific binding. The cells were treated with antibodies against $\mathrm{N}$-cadherin (Abcam, Cambridgeshire, UK), then incubated with a secondary Fluor594 -conjugated goat anti-mouse IgG (Jackson, 1:100 dilution) antibody, and post-stained with DAPI. Fluorescence microscopy was used to photograph the fluorescence images.

\section{Detection of Cy3-labelled miR-106b-5p transfer}

Melanoma cells were transfected with Cy3-labelled miR106b-5p (GenePharma, Shanghai, China) or miR-106b$5 p$ without Cy3-labelling. Then, HEMa-LP cells were cultured with exosomes $(10 \mu \mathrm{g}$ of exosomes resuspended in $100 \mu \mathrm{l}$ PBS) purified from the above mentioned treatment groups. HEMa-LP cells were stained with PKH67 
and DAPI, and images were acquired with a confocal microscope.

\section{Cell invasion, migration and adhesion assays}

For the transwell assay, HEMa-LP cells were resuspended in serum-free DMEM and placed on top of Matrigel-coated chambers (BD Biosciences, USA). DMEM containing 10\% foetal bovine serum was added to the lower chamber as a chemoattractant. After $24 \mathrm{~h}$, we used cotton swabs to remove non-invasive cells. The invasive cells were fixed, stained with $0.1 \%$ crystal violet, counted and imaged using a microscope (100× magnification). For the scratch wound assay, HEMa-LP cells were seeded into 6-well plates, and a $200 \mu$ pipette tip was used to form wound gaps after transfection for $24 \mathrm{~h}$. At 0 and $48 \mathrm{~h}$, the cells were imaged under a microscope to analyse the wound width. ImageJ software was used for imaging analysis. For the adhesion assay, the 96-well plates were pre-coated with fibronectin $(100 \mu \mathrm{g} / \mathrm{ml}$, Sigma, USA) overnight at $4{ }^{\circ} \mathrm{C}$. Plates were blocked with $1 \%$ BSA for an additional $2 \mathrm{~h}$ at $37^{\circ} \mathrm{C}$. A total of $2 \times 10^{4}$ HEMa-LP cells were added to each well and incubated for $40 \mathrm{~min}$. Non-adhesive cells were then removed using PBS. The adhesive cells were fixed and stained with $0.1 \%$ crystal violet, and counted and photographed using a microscope.

\section{Fluorescence in Situ Hybridization (FISH)}

The expression of miR-106b-5p in 5 human malignant melanoma samples and adjacent normal tissues was analysed by FISH. FISH was carried out by using a RiboTM Fluorescent In Situ Hybridization Kit (RiboBio, China) as previously described [23]. Nuclear signals and all signals in the positive sites of the miR-106b-5p probes were counted in different fields of view of the tissue, and 3 fields of view were selected for each group. The FISH results were calculated as follows: FISH result=probe positive signal / nuclear signal. ImageJ software was used to collect the signals.

\section{In vivo pulmonary tumour metastasis assay}

Mice were purchased from the Beijing Laboratory Animal Center (Beijing, China). A375 $\left(1 \times 10^{6}\right.$ cells in plain DMEM) cells stably transfected with the control lentiviral vector or miR-106b-5p inhibitor vector were injected into the tail vein of the mice. The mice were randomly assigned into groups and injected intravenously with $20 \mu \mathrm{g}$ of exosomes secreted by miR-NC- or anti-miR-106b-5p-transfected A375 cells 3 times at $48 \mathrm{~h}$ intervals. miR-106b-5p inhibitor lentiviral vectortransfected A375 cells were injected via the tail vein after the exosome treatment. The mice were anaesthetized before an intraperitoneal injection of sterile D-luciferin Firefly potassium salt solution $(30 \mathrm{mg} / \mathrm{mL})$ after 4 weeks.
Tumour cells colonized in the lung were identified by bioluminescent signals, which were obtained using a Xenogen IVIS 200 instrument (Xenogen, CA) for $4 \mathrm{~min}$ of in vivo imaging. The results were quantified as the average radiance of photons emitted per second and area by using Living Image software (Xenogen, CA). The mice were sacrificed. Then, the lungs were dissected, and the metastatic nodules were counted. This study was approved by the Experimental Animal Ethics Committee of the Affiliated People's Hospital of Jiangsu University.

\section{Immunohistochemistry staining and hematoxylin-eosin (HE) staining}

Immunohistochemistry staining was performed as described previously [24] using antibody against EphA4 (Abcam, Cambridgeshire, UK). For HE staining, sections were incubated with hematoxylin solution after deparaffinization and rehydration. The sections were stained with five dips in $1 \%$ acid ethanol and eosin solution. Graded alcohol was used to dehydrate the sections followed by clearing in xylene. The representative images were taken by fluorescence microscope.

\section{Statistical analysis}

The data are expressed as the mean \pm standard deviation (S.D.), and SPSS 13.0 (IBM, NY, USA) was used to analyze the data. A t-test or one-way ANOVA was used to evaluate statistical significance. Spearman correlation analysis was carried out using MATLAB, and survival plots were drawn using Kaplan-Meier analysis. $P<0.05$ was considered to have statistical significance, and $P<0.01$ had strongly significant.

\section{Results}

miR-106b-5p expression is upregulated in melanoma and an independent risk factor for the survival of patients with melanoma.

First, we analysed miR-106b-5p expression levels in 36 malignant melanoma tissues and adjacent normal tissues by using qRT-PCR. We identified that miR-106b-5p expression was increased in melanoma tissue compared to matched normal tissues (Fig. 1a). The same results were confirmed by analysing previously published datasets (GEO\#GSE34460 and GEO\#GSE24996) (Fig. 1b and c). Moreover, FISH analysis showed a higher miR-106b-5p expression level in 5 selected melanoma tissues than in adjacent normal tissues (Fig. 1d). Malignant melanoma cells (A375, A2058, SK-MEL-1 and SK-MEL-28 cells) expressed higher miR-106b-5p levels than human epidermal melanocytes (HEMa-LP cells) (Fig. 14). Kaplan-Meier analysis showed that patients with melanoma with high miR-106b-5p expression (expression ratio $\geq$ median ratio) had poorer survival (Fig. 1f). 


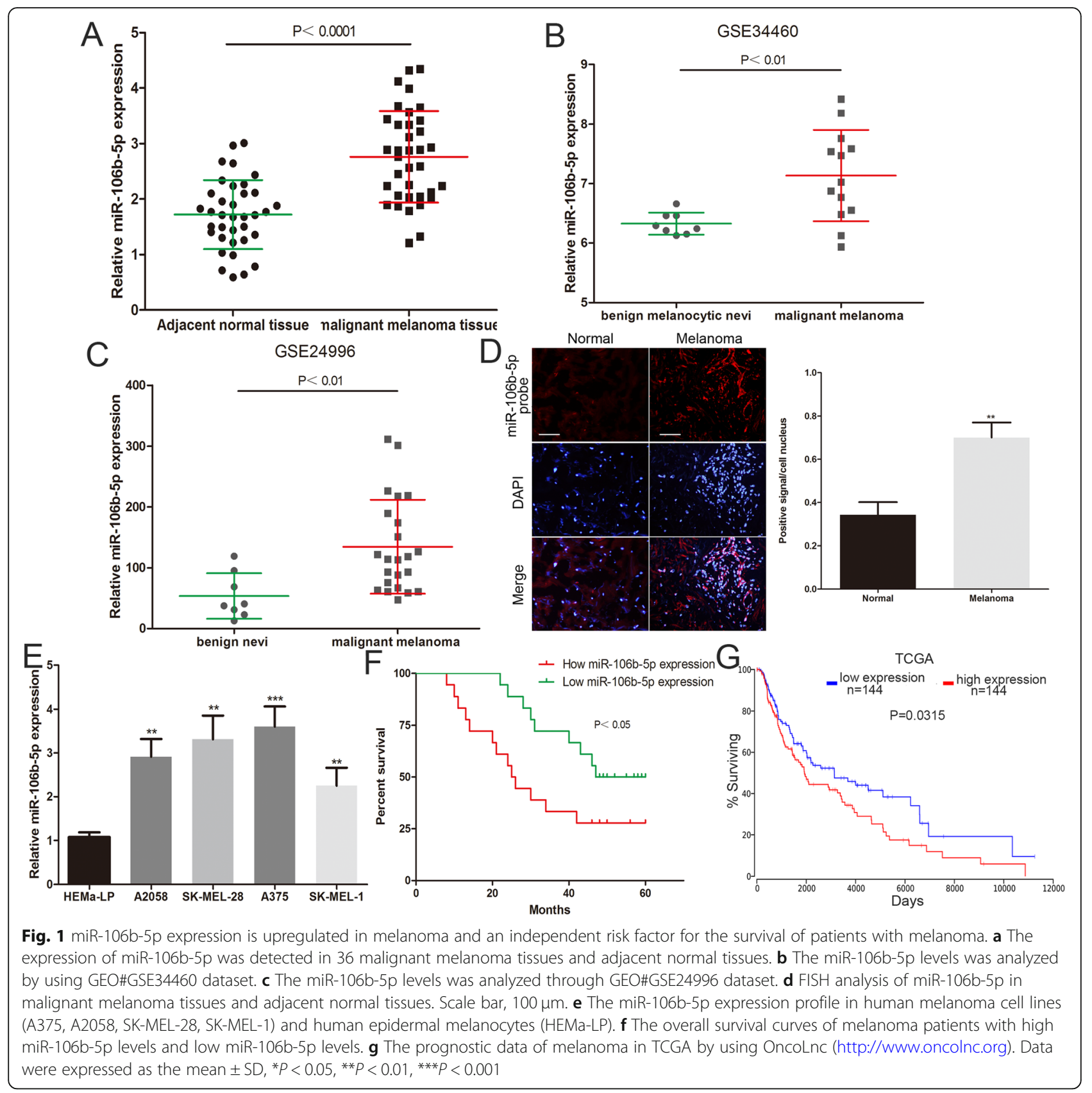

We also analyzed the prognostic data of melanoma in TCGA by using OncoLnc (http://www.oncolnc.org), and found that the survival of melanoma patients with high miR-106b-5p expression ( $n=144$, miR106b-5p expression accounted for the top $33 \%$ of 438 samples) was poorer than the low miR-106b-5p expression group $(n=144$, miR-106b-5p expression accounted for the last 33\% of 438 samples) (Fig. 1g). Furthermore, a high miR-106b-5p expression level was shown to be related to the clinical stage of melanoma but not to the age, sex, family history and occurrence of ulcers (Table 1). Univariate and multivariate Cox regression analyses confirmed that high miR-106b-5p expression is an independent risk factor for the overall survival of patients with melanoma (Table 2). These results suggested that miR106b-5p may be involved in the malignant progression of melanoma.

miR-106b-5p is enriched in melanoma cell-secreted exosomes and transferred to melanocytes

Previous studies have suggested that abundant miRNAs encapsulated in exosomes and play an important role in cell-to-cell communication $[25,26]$. In 
Table 1 Correlation between miR-106b-5p expression and clinical pathological characteristic $(n=36)$

\begin{tabular}{|c|c|c|c|c|}
\hline $\begin{array}{l}\text { Clinical } \\
\text { characteristics }\end{array}$ & Number & $\begin{array}{l}\text { High miR-106b- } \\
5 p \text { expression }\end{array}$ & $\begin{array}{l}\text { Low miR-106b- } \\
5 p \text { expression }\end{array}$ & $P$-value \\
\hline Age & & & & 0.729 \\
\hline$<50$ & 13 & 7 & 6 & \\
\hline$\geq 50$ & 23 & 11 & 12 & \\
\hline Gender & & & & 0.310 \\
\hline Male & 21 & 12 & 9 & \\
\hline Female & 15 & 6 & 9 & \\
\hline Family history & & & & 0.371 \\
\hline Yes & 6 & 2 & 4 & \\
\hline No & 30 & 16 & 14 & \\
\hline TMN stage & & & & $<0.01$ \\
\hline I-II & 14 & 3 & 11 & \\
\hline III & 22 & 15 & 7 & \\
\hline Ulcer & & & & 0.502 \\
\hline Yes & 20 & 9 & 11 & \\
\hline No & 16 & 9 & 7 & \\
\hline
\end{tabular}

addition, serum exosomal miR-106b-5p expression levels were increased in patients with melanoma [16]. Therefore, we investigated whether melanoma cellderived exosomal miR-106b-5p is involved in the malignant progression of melanoma. Exosomes were initially isolated from two malignant melanoma cell lines (A375 and SK-MEL-28 cells). As shown in Fig. 2a, transmission electron microscopy (TEM) showed that exosomes had a typical cup-shaped morphology and were approximately $30-100 \mathrm{~nm}$ in diameter. Western blotting showed the presence of positive exosomal markers (CD63 and TSG101) in both the exosomal and cell fractions (Fig. 2b). Calnexin was used as a negative control, which was confirmed to be absent in the exosomes but present in the cells [27] (Fig. 2b). PKH67 (green) and 4',6diamidino-2-phenylindole (DAPI) (blue) were used to label the exosomes and cell nuclei, respectively. HEMa-LP human epidermal melanocytes were cocultured with purified exosomes. PKH67-labelled exosomes were dispersed in the cytoplasm of HEMa-LP cells (Fig. 2c).

Furthermore, we found that miR-106b-5p was enriched in melanoma cell-secreted exosomes relative to their cellular content (Fig. 2d). The level of exosomal miR-106b-5p expression did not change upon RNaseA treatment but decreased after treatment with RNaseA and Triton X-100, indicating that extracellular miR106b-5p was encapsulated (Fig. 2e). To confirm that melanoma cell-secreted exosomal miR-106b-5p can be transferred to HEMa-LP cells, we analysed the miR106b-5p expression levels in HEMa-LP cells. The cellular expression levels of miR-106b-5p were increased in HEMa-LP cells following the treatment with melanoma cell-secreted exosomes (Fig. 2f). We transfected A375 and SK-MEL-28 cells with Cy3-labelled miR-106b-5p (red), and exosomes from A375 and SK-MEL-28 cells were then isolated and added to HEMa-LP cell culture supernatant. Red fluorescent signals were observed in the Cy3-labelled group but not in the negative control group (Fig. 2g). Overall, these results demonstrated that exosomal miR-106b-5p can be transferred from melanoma cells to HEMa-LP cells, leading to a significant increase in miR-106b-5p expression levels.

\section{Melanoma cell-secreted exosomal miR-106b-5p promotes the EMT of melanocytes}

To further investigate the role of exosomal miR-106b-5p in melanocytes, a lentiviral vector silencing miR-106b-5p or NC was transfected into A375 and SK-MEL-28 cells. Cellular and exosomal miR-106b-5p expression levels were significantly lower in anti-miR-106b-5p-transfected A375 and SK-MEL-28 cells compared with miR-NC-transfected A375 and SK-MEL-28 cells (Fig. 3a). HEMa-LP cells were incubated with exosomes secreted by miR-NC- and anti-miR-106b-5ptransfected melanoma cells. The expression levels of miR-106b-5p in HEMa-LP cells were increased in the miR-NC group, but there was no significant change in the anti-miR-106b-5p group (Fig. 3b). The expression level of the epithelial protein (E-cadherin) was decreased, whereas the mesenchymal proteins (N-cadherin and fibronectin) and EMT-TF (Snail) were increased in HEMa-LP cells incubated with exosomes secreted by miR-NC-transfected melanoma cells (Fig. 3c). Immunofluorescence staining of $\mathrm{N}$-cadherin also showed stronger expression in HEMa-LP cells after miR-NC-transfected melanoma cell exosomes were added (Fig. 3d). The Transwell and scratch wound assays showed that exosomes secreted by miR-NC-transfected melanoma cells promoted

Table 2 Univariate and Multivariate Cox regression analysis of miR-106b-5p associated with overall survival rate in melanoma patients

\begin{tabular}{llll}
\hline Univariate analysis & Hazard ratio & $95 \% \mathrm{Cl}$ & $P$-value \\
miR-106b-5p expression (high vs. low) & 2.517 & $1.057-5.992$ & 0.037 \\
Multivariate analysis & Hazard ratio & $95 \% \mathrm{Cl}$ & $\boldsymbol{P}$-value \\
miR-106b-5p expression (high vs. low) & 2.403 & $1.020-5.658$ & 0.045 \\
\hline
\end{tabular}




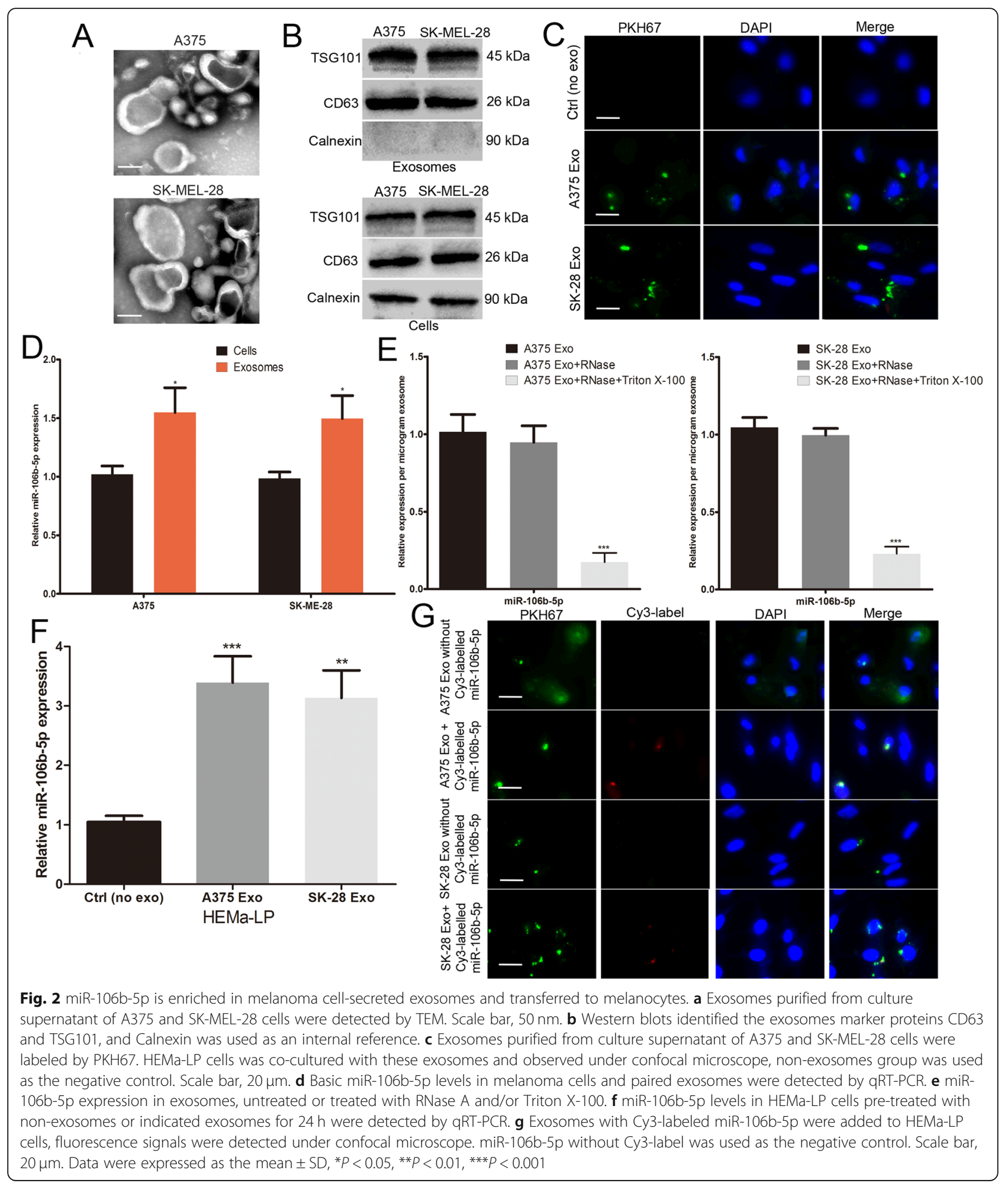

the invasive and migratory ability of HEMa-LP cells (Fig. 3e and f). We also demonstrated that miR-NC-transfected melanoma cell-derived exosomes enhanced HEMa-LP cell adhesion to fibronectin (Fig. 3g). However, exosomes secreted by anti-miR-106b-5p-transfected A375 and SK-MEL-28 cells had no significant effect on the EMT, migration, invasion and adhesion of HEMa-LP cells (Fig. 3c-g). Overall, these data showed 


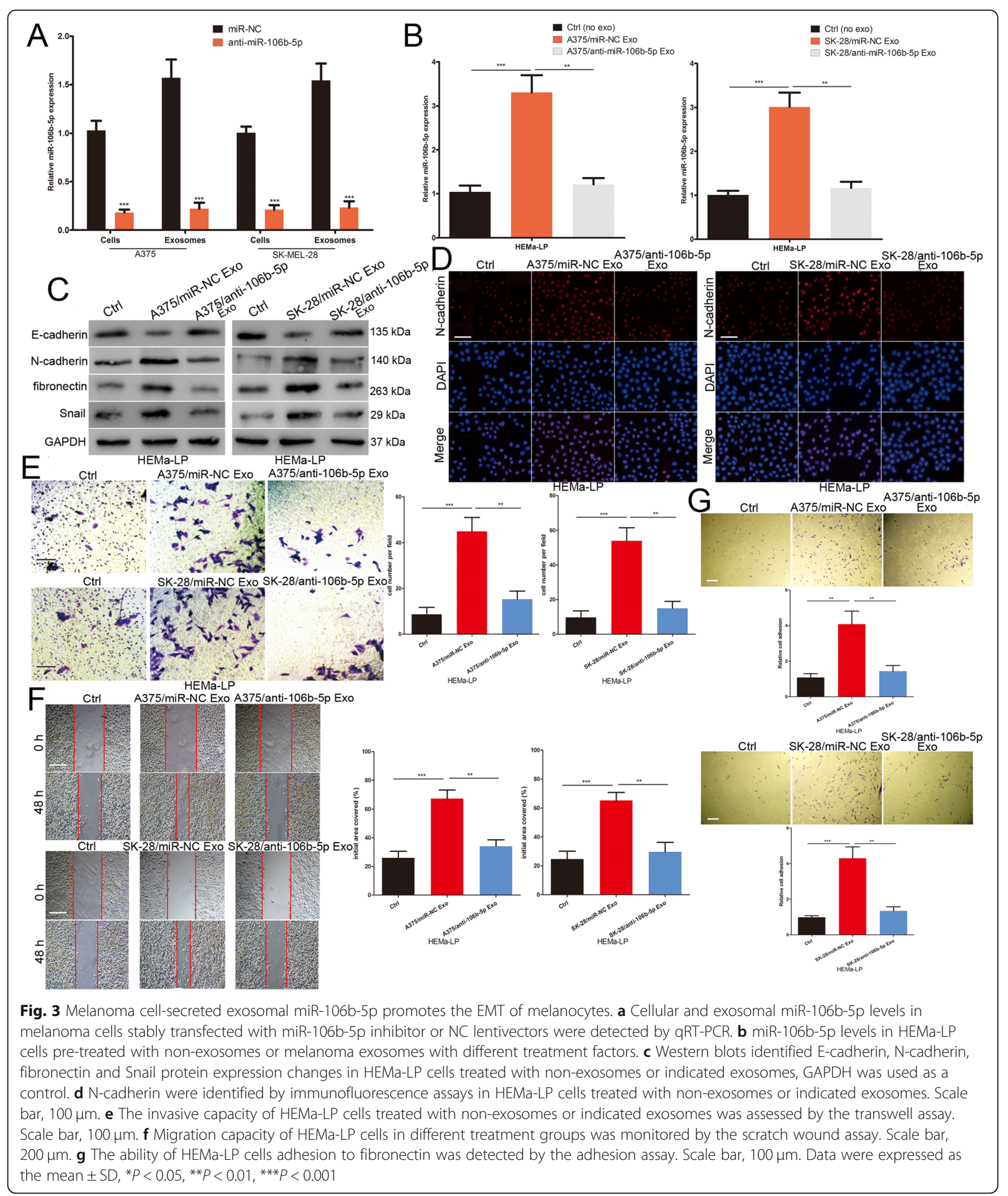

that melanoma cell-derived exosomal miR-106b-5p enhances melanocyte EMT, migration, invasion and adhesion.
EphA4 is a direct target of miR-106b-5p

To investigate how miR-106b-5p exerts its function in melanocytes, we used bioinformatics software to predict 
potential target genes. As predicted by miRNApath, TargetScan, miRDIP, miRanda and miRDB, 464 genes were found to have possible targets with miR-106b-5p (Fig. 4a). We narrowed the miR-106b-5p target genes to 56 genes by overlapping these candidates with genes that are negatively correlated with miR-106b-5p in the TCGA database (Fig. 4b). Among these candidates, EphA4 was shown to function as a negative regulator of the EMT and metastasis of melanoma cells and was chosen for further study [21]. We also verified the negative role of EphA4 in migration, invasion and adhesion of HEMa-LP cells (Supplementary Fig. 1A-C). We constructed EphA4 luciferase reporter vectors containing wild-type and mutant miR-106b-5p binding sites (Fig. $4 c)$. The miR-106b-5p mimic led to a marked decrease in the luciferase activity of the wild-type EphA4 3'UTR plasmid relative to the mutant vector in HEMa-LP cells (Fig. 4d). We used RNA-ChIP analysis to analyse EphA4 mRNA abundance in the Ago2/RNA-induced silencing complex (RISC) after miR-106b-5p overexpression (Fig. $4 \mathrm{e})$. Enrichment in the expression level of miR-106b-5p and EphA4 that was incorporated into RISC were observed in HEMa-LP cells with miR-106b-5p overexpression using RT-qPCR (Fig. 4e). The miR-106b-5p mimic and exosomes derived from the miR-NC-transfected A375 and SK-MEL-28 cells but not from the anti-miR106b-5p-transfected A375 and SK-MEL-28 cells resulted in a decrease in EphA4 expression in HEMa-LP cells at the RNA and protein levels (Fig. $4 \mathrm{f}$ and g). Furthermore, melanoma cells (A375, A2058, SK-MEL-28 and SKMEL-28) expressed lower EphA4 levels compared to human epidermal melanocytes (HEMa-LP) (Fig. 4h). Spearman's correlation between the miR-106b-5p and EphA4 mRNA levels was negative in 36 melanoma tissues (Fig. 4i). TCGA datasets also revealed a statistically significant inverse correlation between miR-106b-5p and EphA4 transcript expression (Fig. 4j). All results indicated that EphA4 is the direct target of miR-106b-5p.

\section{Exosomal miR-106b-5p promotes the EMT of melanocytes by targeting EphA4 to activate the ERK pathway}

EphA4 could interfere with the activation of ERK, which is a major pathway that mediates melanoma cell migration and invasion [21, 28]. We found that exosomes with high miR-106b-5p expression levels led to increased phosphorylated ERK expression in HEMa-LP cells (Fig. 5a). Furthermore, we explored whether exosomal miR-106b-5p induces melanocyte EMT by mediating EphA4 expression. We re-expressed EphA4 in HEMa-LP cells incubated with miR-NC-transfected A375 and SKMEL-28 cell-derived exosomes and silenced EphA4 expression in HEMa-LP cells incubated with anti-miR-106b5p-transfected A375 and SK-MEL-28 cell-derived exosomes (Fig. 5a). The EphA4 plasmid abrogated miR-NC- derived A375 and SK-MEL-28 cell-derived exosomemediated promotion of the EMT, migration, invasion, adhesion and ERK pathway activation of HEMa-LP cells (Fig. 5a-e). EphA4 siRNA could provoke the abilities of anti-miR-106b-5p-transfected A375 and SK-MEL-28 cellderived exosomes to promote the EMT, migration, invasion, adhesion and ERK pathway activation of HEMa-LP cells (Fig. 5a-e). Collectively, these results indicated that exosomal miR-106b-5p induces the EMT of melanocytes by regulating the EphA4/ERK pathway.

\section{Exosomal miR-106b-5p promotes melanoma metastasis in vivo}

We investigated the effect of miR-106b-5p on the invasive ability of melanoma cells in vivo. A375 cells transfected with the NC inhibitor lentivirus or miR-106b-5p inhibitor lentivirus were injected via the tail vein into nude mice (Fig. 6a). miR-106b-5p inhibitor-transfected A375 cells showed less lung colonization than the control group at 2 weeks post-injection (Fig. 6b). Knockdown of miR-106b$5 p$ decreased the number of metastatic lung nodules (Fig. 6c). Nude mice were injected intravenously with $20 \mu \mathrm{g}$ of exosomes secreted by miR-NC- or anti-miR-106b-5ptransfected A375 cells 3 times at $48 \mathrm{~h}$ intervals (Fig. 6a). miR-106b-5p inhibitor-transfected A375 cells were injected via the tail vein after exosome treatment (Fig. 6a). Mice preconditioned with intravenous miR-NCtransfected A375 cells-secreted exosome injections prior to the tail vein inoculation with the miR-106b-5p inhibitor-transfected A375 cells also showed a significant metastatic burden (Fig. $6 \mathrm{~b}$ and c). Tumour tissues from metastatic lung nodules were confirmed by HE staining (Fig. 6d). We analysed relevant signalling molecule expression on sections of metastatic lung nodules. miR-106b-5p expression was increased and EphA4 expression was reduced in the NC inhibitor-transfected group and in the group pre-treated with exosomes secreted by miR-NCtransfected A375 cells (Fig. 6e). We also found that the overexpression of miR-106b-5p was accompanied by activation of ERK pathway (Fig. 6f).

It has been reported that serum exosomal miR$106 \mathrm{~b}-5 \mathrm{p}$ is expressed at significantly higher levels in patients with melanoma [16]. We found that the circulating exosomal miR-106b-5p expression level was positively correlated with tumour miR-106b-5p expression levels but negatively correlated with EphA4 expression (Fig. 6g). FISH analysis and IHC staining also showed that high expression levels of circulating exosomal miR-106b-5p were accompanied by high expression levels of tumour miR-106b-5p and reduced EphA4 expression (Fig. 6h). We finally detected the circulating exosomal miR-106b-5p level in primary and 12 metastatic melanoma patients, 7 of whom had pulmonary metastasis. The clinical characteristic of 


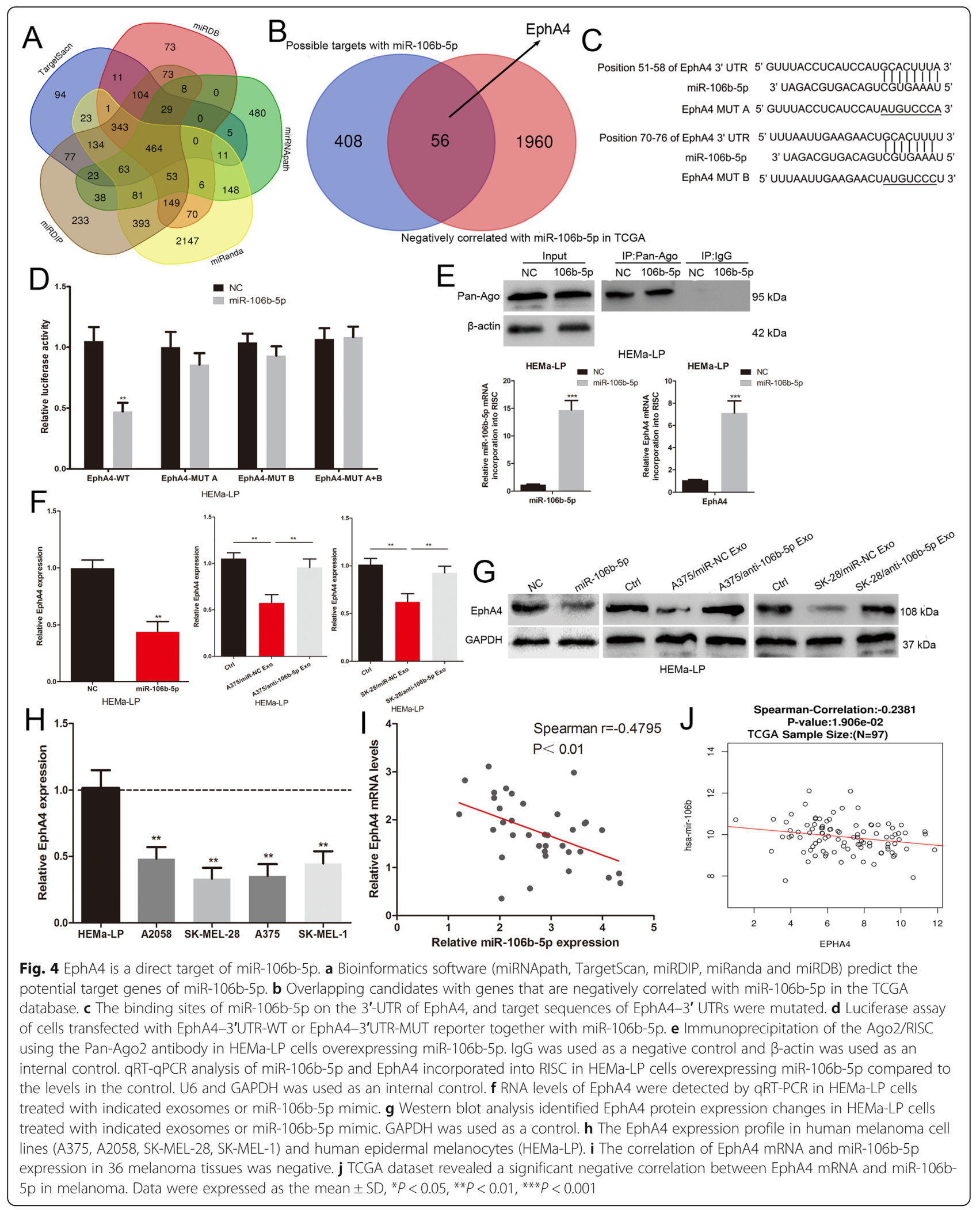




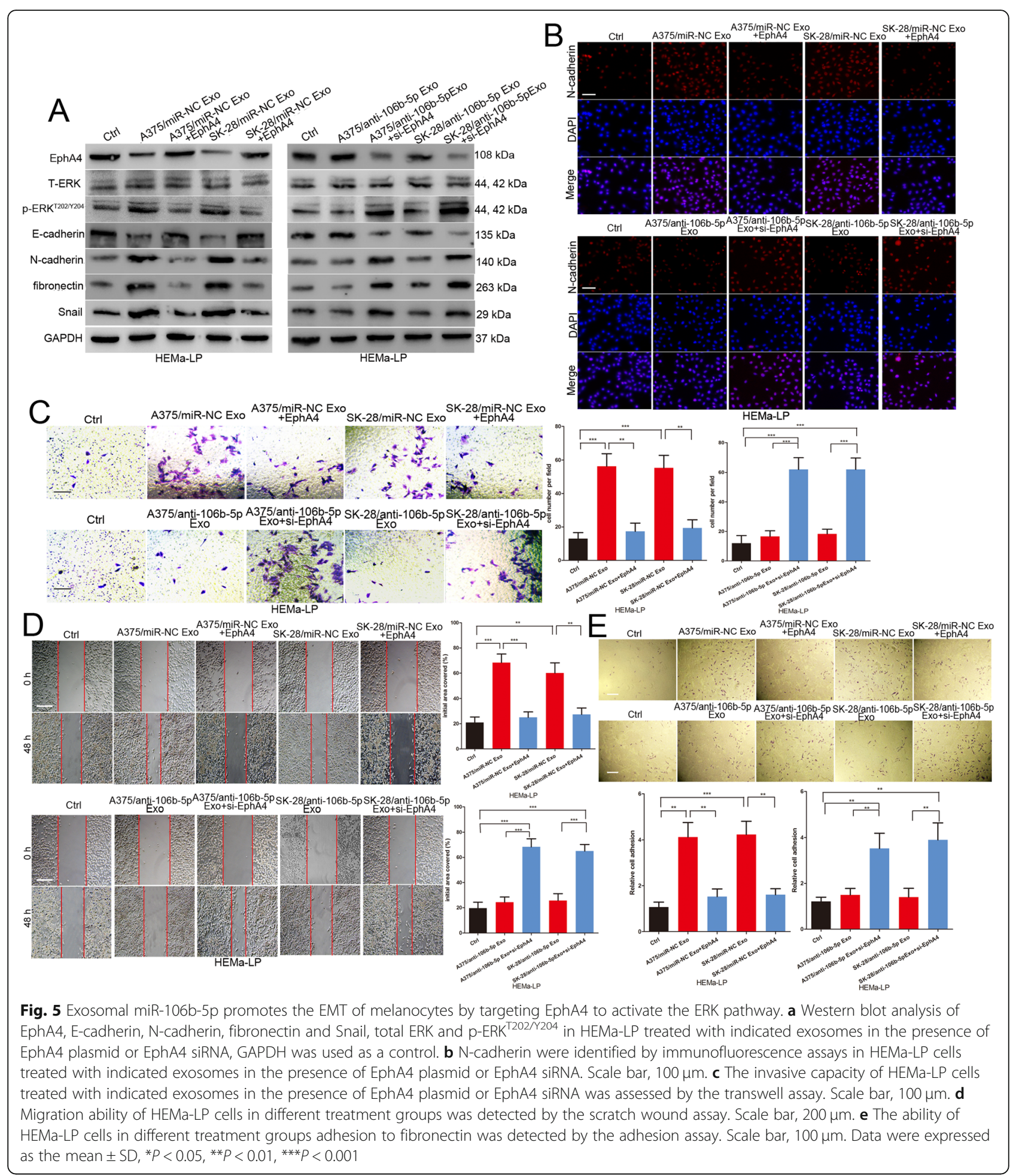

primary and metastatic malignant melanoma was showed in Table 3. The expression of circulating exosomal miR-106b-5p was increased in metastatic melanoma patients compared to primary melanoma patients, the same is true for patients with pulmonary metastases (Fig. $6 \mathrm{i}$ and j). These observations indicated that melanoma cell-secreted exosomal miR106b-5p facilitates melanoma metastasis in vivo.

\section{Discussion}

Melanoma has a high tendency for early metastasis. EMT has implications for tumour cell invasion by 


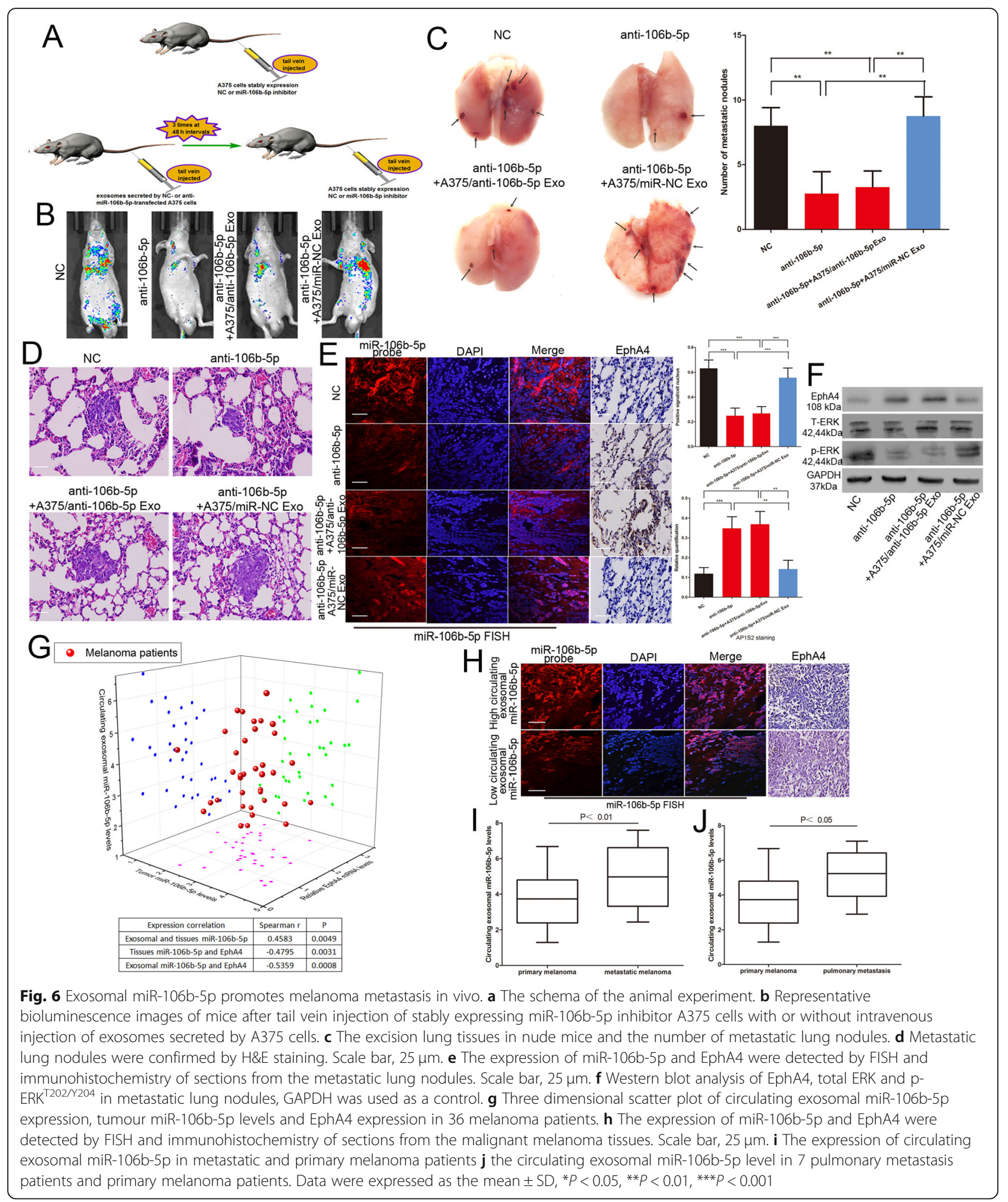

triggering the loss of cell-cell adhesion [29, 30]. Melanocytes express E-cadherin even though they are not epithelial cells [17]. The transformation of melanocytes to melanoma cells involves a series of genetic and environmental changes, and the loss of E-cadherin is the most important change [31]. Many studies have reported that EMT-like processes contribute to melanoma metastasis $[17,18]$. Tumour cell-derived exosomes can carry 
Table 3 The clinical characteristic of primary and metastatic malignant melanoma $(n=48)$

\begin{tabular}{|c|c|c|c|c|}
\hline Clinical characteristics & Number & $\begin{array}{l}\text { Primary malignant } \\
\text { melanoma }(n=36)\end{array}$ & $\begin{array}{l}\text { Metastatic malignant } \\
\text { melanoma }(n=12)\end{array}$ & $P$-value \\
\hline Age & & & & 0.724 \\
\hline$<50$ & 16 & 13 & 3 & \\
\hline$\geq 50$ & 32 & 23 & 9 & \\
\hline Gender & & & & 0.735 \\
\hline Male & 28 & 21 & 7 & \\
\hline Female & 20 & 15 & 5 & \\
\hline Family history & & & & 0.658 \\
\hline Yes & 8 & 6 & 2 & \\
\hline No & 40 & 30 & 10 & \\
\hline
\end{tabular}

oncogenic molecules, which can transfer to normal recipient cells and promote precancerous transformation $[18,32,33]$. Tumour-derived exosomes act as tumour messengers for intercellular crosstalk in the microenvironment and promote the growth and progression of tumours. For instance, breast cancer-derived exosomes induce a myofibroblast phenotype [34]. Exosomes derived from gastric cancer promotes peritoneal metastasis via mesothelial-to-mesenchymal transition [35]. Exosomes also play an important role in the malignant progression of melanoma [36, 37], but there are few studies reporting melanoma cell exosomes inducing EMT in the tumour microenvironment. In this study, we isolated exosomes from two malignant melanoma cell lines and analysed them by TEM and Western blot. Melanocytes acquire EMT characteristics after communicating with melanoma cell-derived exosomes.

Some specific miRNAs could be transferred into recipient cells through cancer cell-secreted exosomes and play a role by reducing the expression of target genes [13]. Liver cancer cell-derived exosomes transfer miR1247-3p to recipient cells and induce CAFs by targeting B4GALT3, eventually leading to lung dissemination [38]. Exosomal miR-221-3p secreted by cervical squamous cell carcinoma promotes lymphatic metastasis by targeting VASH1 [39]. Only a few exosomal miRNAs have been identified as playing a role in the development of melanoma [36, 40]. The expression levels of exosomal miRNAs in the plasma of patients with melanoma are very different, and serum exosomal miR-106b-5p expression is significantly higher in patients with melanoma [16]. However, the function and molecular mechanism of exosomal miR-106b-5p in melanoma remains unknown. Our findings demonstrated that miR-106b-5p expression is upregulated in melanoma tissues and that high miR-106b-5p expression levels are an independent risk factor for patients with melanoma. miR-106b-5p is enriched in melanoma cell-secreted exosomes and transferred to melanocytes. Melanoma cell-derived exosomal
miR-106b-5p expression promotes the EMT, migration, invasion and adhesion of melanocytes.

EphA4, a member of the Eph receptor tyrosine kinase family, has been shown to play different roles in different human tumours. For instance, EphA4 promotes cell proliferation and migration of glioma cells through the FGFR1 signalling pathway [19]. In contrast, EphA4 reduces tumour cell migration and invasion in lung adenocarcinoma through EphA4-dependent ERK1/2 inactivation [20]. Moreover, EPHA4 has been reported to inhibit the EMT and metastasis of melanoma cells by interfering with ERK activation [21]. In this study, we demonstrated that EphA4 is targeted by miR-106b-5p. Exosomal miR-106b-5p promotes the EMT of melanocytes by repressing EphA4 to activate the ERK pathway. We also demonstrated that melanoma cell-secreted exosomal miR-106b-5p facilitates melanoma metastasis in vivo, but the results need further determination in a larger number of samples. In the future, we will collect more clinical samples of metastatic melanoma to further support our findings.

\section{Conclusion}

In summary, the overexpression of miR-106b-5p may play a crucial role in the progression of melanoma. Furthermore, melanoma cell-derived exosomes transfer miR106b-5p to melanocytes. miR-106b-5p targets the EphA4 to activate the ERK pathway and induces the EMT of melanocytes, established the microenvironment characteristics that facilitate tumor metastasis. Understanding the molecular biological basis of tumour-derived exosomes harbouring EMT regulators in melanoma is helpful to recognize new biomarkers and new potential therapeutics for melanoma.

\section{Abbreviations}

qRT-PCR: Quantitative real-time polymerase chain reaction; TEM: Transmission electron microscopy; ChIP: Chromatin Immunoprecipitation; EMT: Epithelialto-mesenchymal transition; EMT-TFs: Epithelial-to-mesenchymal transition inducing transcription factors; EphA4: Erythropoietin-producing 
hepatocellular carcinoma receptor A4; FBS: Foetal bovine serum; GEO: Gene Expression Omnibus; TCGA: The Cancer Genome Atlas; DMEM: Dulbecco's modified Eagle's medium; siRNAs: Small interference RNAs; RT: Reverse transcription; PBS: Phosphate-buffered saline; IF: Immunofluorescence; FISH: Fluorescence in situ hybridization; S.D.: Standard deviation; DAPI: 4',6diamidino-2-phenylindole; HE: Hematoxylin-eosin

\section{Supplementary Information}

The online version contains supplementary material available at https://doi. org/10.1186/s13046-021-01906-w.

\section{Additional file 1: Supplementary Figure 1. The role of EphA4 in} melanocytes. (A) The invasive capacity of HEMa-LP cells was assessed by transwell assay. (B) Migration capacity of HEMa-LP cells in different treatment groups was monitored by scratch wound assay. (C) The ability of HEMa-LP cells adhesion to fibronectin was detected by adhesion assay. Data were expressed as the mean $\pm \mathrm{SD},{ }^{*} P<0.05,{ }^{*} P<0.01,{ }^{* * *} P<0.001$

\section{Acknowledgements}

Not applicable.

\section{Authors' contributions}

WKL conceived and designed the experiments; YTD, HLX, SJM, HRR, JLW and FL performed the experiments. WKL provided the technical support. WKL, HLX and YTD analyzed and interpreted the data. WKL, HLX and YTD wrote the manuscript. The author(s) read and approved the final manuscript.

\section{Funding}

This study was funded by National Natural Science Foundation of China (81802726), Zhenjiang Social Development Foundation of Zhenjiang key R \& D projects (SH2020044).

\section{Availability of data and materials}

All the data and materials supporting the conclusions were included in the main paper.

\section{Declarations}

\section{Ethics approval and consent to participate}

The study was conducted in accordance with the Declaration of Helsinki principles. It was approved by the Ethics Committee of the Affiliated People's Hospital of Jiangsu University.

\section{Consent for publication}

Not applicable.

\section{Competing interests}

The authors declare that they have no competing interests.

\section{Author details \\ ${ }^{1}$ Department of Plastic Surgery, Affiliated People's Hospital of Jiangsu University, 8 Dianli Road, Zhenjiang 212000, Jiangsu, China. ${ }^{2}$ Department of Rehabilitation, Changshu No. 2 People's Hospital (The 5th Clinical Medical College of Yangzhou University), Changshu, Jiangsu, China. ${ }^{3}$ Department of Ophthalmology, Affiliated People's Hospital of Jiangsu University, Zhenjiang, Jiangsu, China.}

Received: 30 November 2020 Accepted: 8 March 2021

Published online: 19 March 2021

\section{References}

1. Siegel RL, Miller KD, Jemal A. Cancer statistics, 2017. CA Cancer J Clin. 2017; 67(1):7-30. https://doi.org/10.3322/caac.21387.

2. Chen W, Zheng $R$, Baade PD, Zhang S, Zeng H, Bray F, Jemal A, Yu XQ, He J. Cancer statistics in China, 2015. CA Cancer J Clin. 2016;66(2):115-32. https:// doi.org/10.3322/caac.21338.

3. Little $E G$, Eide MJ. Update on the current state of melanoma incidence. Dermatol Clin. 2012;30(3):355-61. https://doi.org/10.1016/j.det.2012.04.001.
4. Haass NK, Schumacher U. Melanoma never says die. Exp Dermatol. 2014 23(7):471-2. https://doi.org/10.1111/exd.12400.

5. Tsao H, Chin L, Garraway LA, Fisher DE. Melanoma: from mutations to medicine. Genes Dev. 2012;26(11):1131-55. https://doi.org/10.1101/gad.191999.112.

6. Vlassov AV, Magdaleno S, Setterquist R, Conrad R. Exosomes: current knowledge of their composition, biological functions, and diagnostic and therapeutic potentials. Biochim Biophys Acta. 1820;2012:940-8.

7. Kalluri R, LeBleu VS. The biology, function, and biomedical applications of exosomes. Science. 2020;367(6478):eaau6977.

8. Pegtel DM, Cosmopoulos K, Thorley-Lawson DA, van Eijndhoven MA, Hopmans ES, Lindenberg $J$, de Gruijl TD, Würdinger T, Middeldorp JM. Functional delivery of viral miRNAs via exosomes. Proc Natl Acad Sci U S A. 2010;107(14):6328-33. https://doi.org/10.1073/pnas.0914843107.

9. Azmi AS, Bao B, Sarkar FH. Exosomes in cancer development, metastasis, and drug resistance: a comprehensive review. Cancer Metastasis Rev. 2013; 32(3-4):623-42. https://doi.org/10.1007/s10555-013-9441-9.

10. Galasso M, Sandhu SK, Volinia S. MicroRNA expression signatures in solid malignancies. Cancer J. 2012;18(3):238-43. https://doi.org/10.1097/PPO. Ob013e318258b5f4.

11. Filipowicz W, Bhattacharyya SN, Sonenberg N. Mechanisms of posttranscriptional regulation by microRNAs: are the answers in sight. Nat Rev Genet. 2008;9(2):102-14. https://doi.org/10.1038/nrg2290.

12. Felicetti F, De Feo A, Coscia C, Puglisi R, Pedini F, Pasquini L, Bellenghi M, Errico MC, Pagani E, Carè A. Exosome-mediated transfer of miR-222 is sufficient to increase tumor malignancy in melanoma. J Transl Med. 2016; 14(1):56. https://doi.org/10.1186/s12967-016-0811-2.

13. Hashimoto K, Ochi H, Sunamura S, Kosaka N, Mabuchi Y, Fukuda T, Yao K, Kanda H, Ae K, Okawa A, Akazawa C, Ochiya T, Futakuchi M, Takeda S, Sato S. Cancer-secreted hsa-miR-940 induces an osteoblastic phenotype in the bone metastatic microenvironment via targeting ARHGAP1 and FAM134A. Proc Natl Acad Sci U S A. 2018;115(9):2204-9. https://doi.org/10.1073/pnas.1 717363115 .

14. Xiao D, Ohlendorf J, Chen Y, Taylor DD, Rai SN, Waigel S, Zacharias W, Hao $\mathrm{H}$, McMasters KM. Identifying mRNA, microRNA and protein profiles of melanoma exosomes. PLoS One. 2012;7(10):e46874. https://doi.org/10.1371/ journal.pone.0046874.

15. Gajos-Michniewicz A, Duechler M, Czyz M. MiRNA in melanoma-derived exosomes. Cancer Lett. 2014;347(1):29-37. https://doi.org/10.1016/..canlet.2 014.02.004.

16. Tengda L, Shuping L, Mingli G, Jie G, Yun L, Weiwei Z, Anmei D. Serum exosomal microRNAs as potent circulating biomarkers for melanoma. Melanoma Res. 2018;28(4):295-303. https://doi.org/10.1097/CMR. 0000000000000450

17. Caramel J, Papadogeorgakis E, Hill L, Browne GJ, Richard G, Wierinckx A, Saldanha G, Osborne J, Hutchinson P, Tse G, Lachuer J, Puisieux A, Pringle $J$, Ansieau S, Tulchinsky E. A switch in the expression of embryonic EMTinducers drives the development of malignant melanoma. Cancer Cell. 2013;24(4):466-80. https://doi.org/10.1016/j.ccr.2013.08.018.

18. Xiao D, Barry S, Kmetz D, Egger M, Pan J, Rai SN, Qu J, McMasters KM, Hao H. Melanoma cell-derived exosomes promote epithelial-mesenchymal transition in primary melanocytes through paracrine/autocrine signaling in the tumor microenvironment. Cancer Lett. 2016;376(2):318-27. https://doi. org/10.1016/j.canlet.2016.03.050.

19. Fukai J, Yokote H, Yamanaka R, Arao T, Nishio K, Itakura T. EphA4 promotes cell proliferation and migration through a novel EphA4-FGFR1 signaling pathway in the human glioma U251 cell line. Mol Cancer Ther. 2008;7(9): 2768-78. https://doi.org/10.1158/1535-7163.MCT-07-2263.

20. Saintigny P, Peng S, Zhang L, Sen B, Wistuba II, Lippman SM, Girard L, Minna JD, Heymach JV, Johnson FM. Global evaluation of Eph receptors and ephrins in lung adenocarcinomas identifies EphA4 as an inhibitor of cell migration and invasion. Mol Cancer Ther. 2012;11(9):2021-32. https://doi. org/10.1158/1535-7163.MCT-12-0030.

21. Hua KT, Hong JB, Sheen YS, Huang HY, Huang YL, Chen JS, Liao YH. miR519d promotes melanoma progression by Downregulating EphA4. Cancer Res. 2018;78(1):216-29. https://doi.org/10.1158/0008-5472.CAN-17-1933.

22. Luan W, Zhou Z, Ni X, Xia Y, Wang J, Yan Y, Xu B. Long non-coding RNA H19 promotes glucose metabolism and cell growth in malignant melanoma via miR-106a-5p/E2F3 axis. J Cancer Res Clin Oncol. 2018;144(3): 531-42. https://doi.org/10.1007/s00432-018-2582-z.

23. Liu H, Dai C, Wu Q, Liu H, Li F. Expression profiling of long noncoding RNA identifies Inc-MMP3-1 as a prognostic biomarker in external auditory canal 
squamous cell carcinoma. Cancer Med. 2017;6(11):2541-51. https://doi.org/1 $0.1002 / \mathrm{cam} 4.1213$

24. Luan W, Wang Y, Chen X, Shi Y, Wang J, Zhang J, Qian J, Li R, Tao T, Wei W, Hu Q, Liu N, You Y. PKM2 promotes glucose metabolism and cell growth in gliomas through a mechanism involving a let-7a/c-Myc/hnRNPA1 feedback loop. Oncotarget. 2015;6(15):13006-18. https://doi.org/10.1 8632/oncotarget.3514.

25. Li L, Li C, Wang S, Wang Z, Jiang J, Wang W, Li X, Chen J, Liu K, Li C, Zhu G. Exosomes derived from hypoxic Oral squamous cell carcinoma cells deliver miR-21 to normoxic cells to elicit a Prometastatic phenotype. Cancer Res. 2016;76(7):1770-80. https://doi.org/10.1158/0008-5472.CAN-15-1625.

26. Valadi H, Ekström K, Bossios A, Sjöstrand M, Lee JJ, Lötvall JO. Exosomemediated transfer of mRNAs and microRNAs is a novel mechanism of genetic exchange between cells. Nat Cell Biol. 2007;9(6):654-9. https://doi. org/10.1038/ncb1596.

27. Gastpar R, Gehrmann M, Bausero MA, Asea A, Gross C, Schroeder JA, Multhoff $\mathrm{G}$. Heat shock protein 70 surface-positive tumor exosomes stimulate migratory and cytolytic activity of natural killer cells. Cancer Res. 2005;65(12):5238-47. https://doi.org/10.1158/0008-5472.CAN-04-3804.

28. Satyamoorthy K, Li G, Gerrero MR, Brose MS, Volpe P, Weber BL, Van Belle P, Elder DE, Herlyn M. Constitutive mitogen-activated protein kinase activation in melanoma is mediated by both BRAF mutations and autocrine growth factor stimulation. Cancer Res. 2003;63(4):756-9.

29. Vella $L J$. The emerging role of exosomes in epithelial-mesenchymaltransition in cancer. Front Oncol. 2014;4:361.

30. Christiansen JJ, Rajasekaran AK. Reassessing epithelial to mesenchymal transition as a prerequisite for carcinoma invasion and metastasis. Cancer Res. 2006;66(17):8319-26. https://doi.org/10.1158/0008-5472.CAN-06-0410.

31. Lee JT, Herlyn M. Microenvironmental influences in melanoma progression. J Cell Biochem. 2007;101(4):862-72. https://doi.org/10.1002/jcb.21204.

32. Grange C, Tapparo M, Collino F, Vitillo L, Damasco C, Deregibus MC, Tetta C, Bussolati B, Camussi G. Microvesicles released from human renal cancer stem cells stimulate angiogenesis and formation of lung premetastatic niche. Cancer Res. 2011;71(15):5346-56. https://doi.org/1 0.1158/0008-5472.CAN-11-0241.

33. Taverna S, Flugy A, Saieva L, Kohn EC, Santoro A, Meraviglia S, De Leo $G$, Alessandro R. Role of exosomes released by chronic myelogenous leukemia cells in angiogenesis. Int J Cancer. 2012;130(9):2033-43. https://doi.org/10.1002/ijc.26217.

34. Cho JA, Park H, Lim EH, Lee KW. Exosomes from breast cancer cells can convert adipose tissue-derived mesenchymal stem cells into myofibroblastlike cells. Int J Oncol. 2012;40(1):130-8. https://doi.org/10.3892/ijo.2011.1193.

35. Li Q, Li B, Li Q, Wei S, He Z, Huang X, Wang L, Xia Y, Xu Z, Li Z, Wang W, Yang L, Zhang D, Xu Z. Exosomal miR-21-5p derived from gastric cancer promotes peritoneal metastasis via mesothelial-to-mesenchymal transition. Cell Death Dis. 2018;9(9):854. https://doi.org/10.1038/s41419-018-0928-8.

36. Li J, Chen J, Wang S, Li P, Zheng C, Zhou X, et al. Blockage of transferred exosome-shuttled miR-494 inhibits melanoma growth and metastasis. J Cell Physiol. 2019.

37. Alegre E, Zubiri L, Perez-Gracia JL, Gonzalez-Cao M, Soria L, Martin-Algarra S, et al. Circulating melanoma exosomes as diagnostic and prognosis biomarkers. Clin Chim Acta. 2016;454:28-32.

38. Fang T, Lv H, Lv G, Li T, Wang C, Han Q, Yu L, Su B, Guo L, Huang S, Cao D, Tang L, Tang S, Wu M, Yang W, Wang H. Tumor-derived exosomal miR1247-3p induces cancer-associated fibroblast activation to foster lung metastasis of liver cancer. Nat Commun. 2018;9(1):191. https://doi.org/10.103 8/s41467-017-02583-0.

39. Zhou CF, Ma J, Huang L, Yi HY, Zhang YM, Wu XG, Yan RM, Liang L, Zhong M, Yu YH, Wu S, Wang W. Cervical squamous cell carcinoma-secreted exosomal miR-221-3p promotes lymphangiogenesis and lymphatic metastasis by targeting VASH1. Oncogene. 2019;38(8):1256-68. https://doi. org/10.1038/s41388-018-0511-X.

40. Zhou X, Yan T, Huang C, Xu Z, Wang L, Jiang E, Wang H, Chen Y, Liu K, Shao Z, Shang Z. Melanoma cell-secreted exosomal miR-155-5p induce proangiogenic switch of cancer-associated fibroblasts via SOCS1/JAK2/ STAT3 signaling pathway. J Experiment Clin Cancer Res : CR. 2018;37(1):242. https://doi.org/10.1186/s13046-018-0911-3.

\section{Publisher's Note}

Springer Nature remains neutral with regard to jurisdictional claims in published maps and institutional affiliations.

Ready to submit your research? Choose BMC and benefit from:

- fast, convenient online submission

- thorough peer review by experienced researchers in your field

- rapid publication on acceptance

- support for research data, including large and complex data types

- gold Open Access which fosters wider collaboration and increased citations

- maximum visibility for your research: over $100 \mathrm{M}$ website views per year

At BMC, research is always in progress.

Learn more biomedcentral.com/submissions 